\title{
Sloshing of liquid in rigid cylindrical container with a rigid annular baffle. Part II: Lateral excitation
}

\author{
J.D. Wang, D. Zhou* and W.Q. Liu \\ College of Civil Engineering, Nanjing University of Technology, Nanjing, Jiangsu, China
}

Received 11 June 2011

Revised 10 November 2011

\begin{abstract}
Sloshing response of liquid in a rigid cylindrical container with a rigid annual baffle subjected to lateral excitation has been studied. The complicated liquid domain is separated into several simple sub-domains by introducing the artificial interfaces. The analytical solutions of potential function corresponding to every sub-domain are obtained by using the method of separation of variables and the superposition principle. The total potential function under lateral excitation is taken as the sum of the container potential function and the liquid perturbed function. The expression of the liquid perturbed function is obtained by introducing the generalized coordinates. On the base of the natural frequencies and modes having been obtained by the sub-domain method, the orthogonality among the sloshing modes has been demonstrated. Substituting the potential functions into the free surface wave equation establishes the dynamic response equation of liquid. Then, the generalized coordinates are solved. The sloshing surface displacement, the hydrodynamic pressure distribution, the resultant hydrodynamic force and moment are discussed for the containers subjected to harmonic and seismic lateral excitation, respectively.
\end{abstract}

Keywords: Cylindrical container, annular baffle, sub-domain, liquid perturbed function, sloshing response, analytical method

\section{Introduction}

The free surface in a partially filled liquid container allows for liquid motion relative to the container. Liquid sloshing is generally caused by external container excitation, and often has significant influence on the response of the container. Numerous investigations can be found on the response of liquid sloshing subjected to external container excitation [1]. The excitation can be impulsive, sinusoidal, periodic, or random. Its orientation with respect to the container can be lateral or pitching.

Under impulsive excitation, liquid sloshing dynamics was studied by Morris [2], Werner and Sundquist [3], Jacobsen [4], Jacobsen and Ayre [5], and Hoskins and Jacobsen [6]. Bauer [7] considered the response of liquid propellant under a single pulse excitation. Transient and steady state response under harmonic excitation of a liquid free surface was studied by Sogabe and Shibata [8,9]. Housner [10] presented the simplified formulae to compute the dynamic pressures developed on accelerated liquid containers. Then Housner [11] studied the dynamic behavior of ground-supported elevated water containers by using the equivalent spring-mass systems. Furthermore, Haroun and Housner [12] presented a simple and sufficiently accurate method to estimate the seismic response of cylindrical liquid storage tanks by introducing a mechanical model in which, the deformability of the tank wall is taken into account. The model is based on the results of a finite element analysis for the liquid-shell system. Meserole and

\footnotetext{
${ }^{*}$ Corresponding author: D. Zhou, College of Civil Engineering, Nanjing University of Technology, Nanjing 210009, Jiangsu, China. E-mail: dingzhou57@yahoo.com.
} 
Fortini [13] investigated the liquid sloshing dynamics in a 1:4 scale model of annular liquid oxygen container for a minimum-length orbit transfer vehicle by the equivalent mechanical models which were derived experimentally and analytically. Abramson and Ransleben [14] discussed and compared some results obtained from sloshing experiments with rigid cylindrical containers in translation, and having both flat and conical bottoms. In addition, the special NASA monograph edited by Abramson [15] for liquid sloshing in aerospace vehicles was published. The monograph contained the analytical and experimental studies of linear and nonlinear sloshing, damping of liquid motions, vertical excitations of containers, interaction of liquid propellants and elastic structures, vehicle stability and control, liquid-propellant behavior at low and zero gravity, longitudinal oscillation of flight vehicles, etc. Aslam et al. [16] studied the linear sloshing response of water in annular and cylindrical containers under horizontal earthquake ground motions by an analytical method and experiments. And then, Aslam [17] developed a finite element formulation based on a linearized wave theory to predict the sloshing displacements and hydrodynamic pressure in an axisymmetric rigid container due to arbitrary ground acceleration.

Bauer [18] determined the response of the liquid in an arbitrary partially filled paraboloidal container under harmonic translational and roll excitation about its axis. Bauer and Eidel [19] considered free and forced oscillations of a frictionless liquid with a free surface in a prolate spheroidal container analytically. A Lagrangian formulation was developed by Budiansky [20] to examine the sloshing dynamics under horizontal excitation of spherical and circular horizontal tanks. Isaacson and Ryu [21] studied the sloshing of liquid in a rigid vertical cylindrical container with arbitrary cross-section under both harmonic and irregular excitation by an eigen-function expansion method in which, a set of boundary integral equations involving a suitable Green's function are solved for each mode. Kobayashi et al. [22] conducted an experimental and analytical study to determine the liquid natural frequencies and the resultant sloshing forces in horizontal cylindrical containers. Papaspyrou et al. [23] developed a mathematical model for calculating liquid sloshing effects such as hydrodynamic pressures and resultant forces in half-full spherical containers under arbitrary external excitation by expressing the velocity potential in a series form. Additionally, Papaspyrou et al. [24] investigated the response of half-full horizontal cylindrical vessels under external excitation in transverse direction by the similar mathematical model.

Most of the published works mentioned above are concerned with the natural frequencies, modes and response of liquid-filled containers without any slosh damping devices. However, baffles are generally used as passive slosh damping devices in the liquid storage containers. A few studies recently reported the effects of baffles on the dynamic response of liquid sloshing in containers. Gedikli and Ergüven [25] analyzed the seismic response of a rigid liquid-filled container with a rigid baffle by the boundary element method. Malelci and Ziyaeifar [26] investigated the damping effect of baffles in reducing the seismic response of isolated cylindrical liquid storage containers. Biswal et al. [27] studied the influence of a baffle on the dynamic response of a partially liquid-filled cylindrical container by the finite element method. Furthermore, Biswal et al. [28] considered the dynamic interaction that exists between the liquid and elastic container-baffle system to evaluate the coupled response of liquid and container-baffle system by the similar method. Based on the zoning method, a boundary element method was developed to determine the natural frequencies and mode shapes of liquid sloshing in 3D baffled tanks with arbitrary geometries by Firouz-Abadi et al. [29]. Furthermore, Firouz-Abadi [30] developed the reduced order modeling technique to investigate liquid sloshing in three dimensional containers using the boundary element method.

To our knowledge, most researches on liquid sloshing of baffled liquid-filled containers are concerned with numerical methods such as finite element method, boundary element method and finite difference method. Many useful and satisfactory results have been provided by these methods. However, these numerical methods depend primarily on the refinement of the meshes and the construction of such precise meshes is usually difficult and time-consuming task. Additionally, elements often become over-distorted during simulating the response of liquid sloshing. Recently, the authors [31] investigated the free vibration of liquid in a rigid cylindrical container with a rigid annular baffle by using the analytical method, which establishes the base of the present study. In this paper, an analytical method is developed to solve the forced sloshing problems in a rigid cylindrical container with a rigid annular baffle under lateral excitation. The effect of the liquid surface waves is taken into account in the analysis. The complicated liquid domain is separated into several simple sub-domains and the total potential function is assumed to the sum of the container potential function and the perturbed function. After substituting the total potential function into the free surface wave conditions, the dynamic response equations under generalized coordinates, which are a series of uncoupled second-order common differential equations about time, are developed. The generalized coordinates can be solved from the dynamic response equations. 


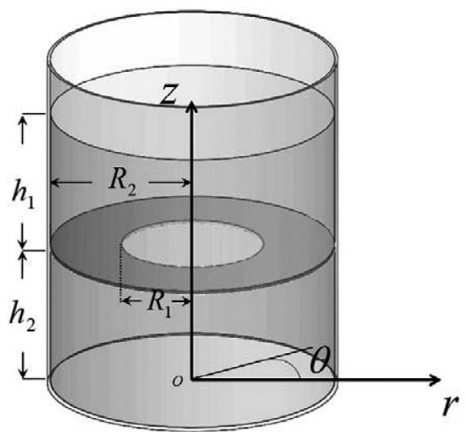

Fig. 1. Container and baffle.

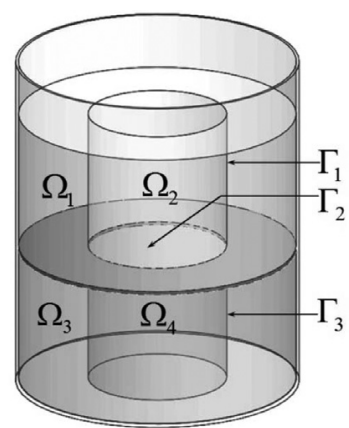

Fig. 2. Sub-domains and interfaces.

\section{Governing equation of liquid movement}

Consider a thin rigid annular baffle placed in a rigid circular cylindrical container partially filled with inviscid, incompressible and irrotational liquid, as shown in Fig. 1. The liquid has a free surface which is orthogonal to axis of the container. Free-surface waves are considered in the present analysis, and a linear theory of liquid movement is adopted from the small-amplitude sloshing of the liquid. The baffle is very thin compared to its diameter and the effect of the thickness can be neglected. The fluid height $h$ is divided into $h_{1}$ and $h_{2}$ where $h_{1}$ is the length between the free surface and the baffle's neutral plane and $h_{2}$ is the length between the baffle's neutral plane and the bottom. The radius of the container is $R_{2}$ and the inside radius of the baffle is $R_{1}$. The cylindrical coordinates are used to describe the movement of the liquid and its origin is located at the center of the bottom of the container. As shown in Fig. 2, the liquid domain $\Omega$ in the cylindrical with an annular baffle can be separated into four sub-domains: $\Omega_{i}$ $(i=1,2,3,4)$, namely

$$
\phi(r, \theta, z, t)=\phi_{i}(r, \theta, z, t),(r, \theta, z) \in \Omega_{i},(i=1,2,3,4)
$$

in which, $\phi_{i}$ is the total potential function of the sub-domain $\Omega_{i}$ and $\phi$ is the total potential function of the liquid domain $\Omega$. According to the above assumptions, the potential function of liquid movement should satisfy the Laplace equation:

$$
\frac{1}{r} \frac{\partial}{\partial r}\left(r \frac{\partial \phi_{i}}{\partial r}\right)+\frac{1}{r^{2}} \frac{\partial^{2} \phi_{i}}{\partial \theta^{2}}+\frac{\partial^{2} \phi_{i}}{\partial z^{2}}=0 \text { in } \Omega_{i}
$$

The relations between $\phi_{i}$ and velocity components of liquid are

$$
v_{r i}=\frac{\partial \phi_{i}}{\partial r}, v_{\theta i}=\frac{\partial \phi_{i}}{r \partial \theta}, v_{z i}=\frac{\partial \phi_{i}}{\partial z}
$$

Assume that $\Omega_{i}$ and $\Omega_{l}(i<l)$ are in contact with each other and their interface is $\Gamma_{k}$. According to Fig. 2, the ordered triple $(i, l, k)$ should belong to the set $\{(1,2,1),(2,4,2),(3,4,3)\}$. Obviously, $\Omega_{i}$ and $\Omega_{l}$ should satisfy the continuous conditions of pressure and velocity on $\Gamma_{k}$, i.e.

$$
\begin{aligned}
& \phi_{i}=\phi_{l} \text { on } \Gamma_{k} \\
& \frac{\partial \phi_{i}}{\partial \vec{\tau}_{k}}=\frac{\partial \phi_{l}}{\partial \vec{\tau}_{k}} \text { on } \Gamma_{k} \\
& \frac{\partial \phi_{i}}{\partial \vec{n}_{k}}=\frac{\partial \phi_{l}}{\partial \vec{n}_{k}} \text { on } \Gamma_{k}
\end{aligned}
$$

in which, $\vec{n}_{k}$ denotes the normal vector of $\Gamma_{k} . \vec{\tau}_{k}$ denotes the tangential vector of $\Gamma_{k}$. It is obvious that Eq. (5) can be easily deduced from Eq. (4). 


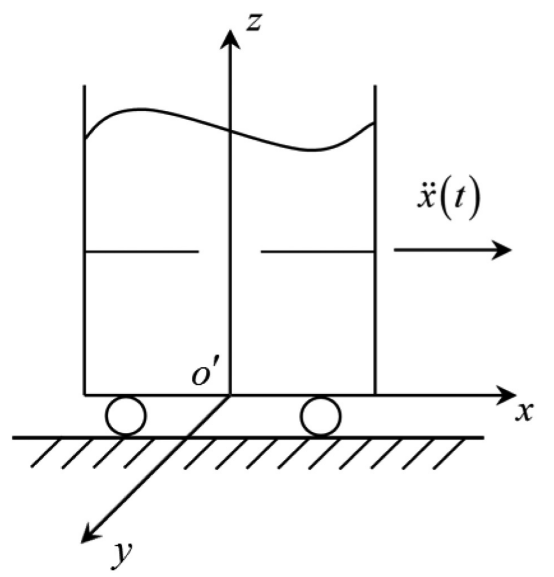

Fig. 3. Container's acceleration.

\section{Boundary equations and initial conditions}

In the present analysis, the liquid hydrodynamic pressure, resultant force and moment acting on the circular cylindrical container with an annular baffle are estimated under lateral excitation. The inertial coordinate system $o^{\prime} x y z$ is given to describe the absolute motion of the container. Consider the container subjected to lateral excitation $x(t)$ along the $x$-direction as shown in Fig. 3. Under the assumption that the amplitudes of excitation and liquid response are small, the linearized boundary equations and initial conditions of the liquid are in the form of

$$
\begin{aligned}
& \left.\frac{\partial \phi_{i}}{\partial \theta}\right|_{\theta=k \pi}=0(i=1,3),\left.\frac{\partial \phi_{i}}{\partial r}\right|_{r=R_{2}}=\dot{x}(t) \cos \theta(i=1,3, k=0,1,2, \cdots) \\
& \left.\frac{\partial \phi_{i}}{\partial z}\right|_{z=h_{2}}=0(i=1,3),\left.\frac{\partial \phi_{i}}{\partial z}\right|_{z=0}=0(i=3,4) \\
& \left.\phi\right|_{t=0}=\phi_{0},\left.\quad \dot{\phi}\right|_{t=0}=\dot{\phi}_{0} \\
& \left.\frac{\partial \phi_{i}}{\partial t}\right|_{z=h}+g f_{i}=0,(i=1,2) \\
& f_{i}=\left.\int_{0}^{t} \frac{\partial \phi_{i}}{\partial z}\right|_{z=h} d t,(i=1,2)
\end{aligned}
$$

in which, $f_{i}$ is the sloshing displacement of the liquid in $\Omega_{i}$. The total potential function $\phi_{i}$ of the sub-domain $\Omega_{i}$ is taken as the sum of the liquid perturbed function $\phi_{i B}(r, \theta, z, t)$ and the container potential function $\phi_{i A}(r, \theta, z, t)$. Equations (4)-(6) can be rewritten in the following form:

$$
\begin{aligned}
& \phi_{i A}=\phi_{l A}, \phi_{i B}=\phi_{l B} \text { on } \Gamma_{k} \\
& \frac{\partial \phi_{i A}}{\partial \vec{\tau}_{k}}=\frac{\partial \phi_{l A}}{\partial \vec{\tau}_{k}}, \frac{\partial \phi_{i B}}{\partial \vec{\tau}_{k}}=\frac{\partial \phi_{l B}}{\partial \vec{\tau}_{k}} \text { on } \Gamma_{k} \\
& \frac{\partial \phi_{i A}}{\partial \vec{n}_{k}}=\frac{\partial \phi_{l A}}{\partial \vec{n}_{k}}, \frac{\partial \phi_{i B}}{\partial \vec{n}_{k}}=\frac{\partial \phi_{l B}}{\partial \vec{n}_{k}} \text { on } \Gamma_{k}
\end{aligned}
$$

According to Eqs (7)-(11), the governing equation and boundary conditions of $\phi_{i A}(r, \theta, z, t)$ and $\phi_{i B}(r, \theta, z, t)$ can be given as 


$$
\begin{aligned}
& \nabla^{2} \phi_{i A}=0,(i=1,2,3,4) \\
& \nabla^{2} \phi_{i B}=0,(i=1,2,3,4) \\
& \left.\frac{\partial \phi_{i A}}{\partial \theta}\right|_{\theta=k \pi}=\left.\frac{\partial \phi_{i B}}{\partial \theta}\right|_{\theta=k \pi}=0,(i=1,3, k=0,1,2, \cdots) \\
& \left.\frac{\partial \phi_{i A}}{\partial r}\right|_{r=R_{2}}=\dot{x}_{0}(t) \cos \theta,(i=1,3) \\
& \left.\frac{\partial \phi_{i B}}{\partial r}\right|_{r=R_{2}}=0,(i=1,3) \\
& \left.\frac{\partial \phi_{i A}}{\partial z}\right|_{z=0}=\left.\frac{\partial \phi_{i B}}{\partial z}\right|_{z=0}=0,(i=3,4) \\
& \left.\frac{\partial \phi_{i A}}{\partial z}\right|_{z=h_{2}}=\left.\frac{\partial \phi_{i B}}{\partial z}\right|_{z=h_{2}}=0,(i=1,3) \\
& \left.\frac{\partial \phi_{i B}}{\partial t}\right|_{z=h}+g f_{i B}=-\left.\frac{\partial \phi_{i A}}{\partial t}\right|_{z=h}-g f_{i A},(i=1,2) \\
& {\left.\left[\phi_{i A}+\phi_{i B}\right]\right|_{t=0}=\phi_{i 0},(i=1,2,3,4)} \\
& {\left.\left[\dot{\phi}_{i A}+\dot{\phi}_{i B}\right]\right|_{t=0}=\dot{\phi}_{i 0},(i=1,2,3,4)} \\
& f_{i A}=\left.\int_{0}^{t} \frac{\partial \phi_{i A}}{\partial z}\right|_{z=h} d t,(i=1,2) \\
& f_{i B}=\left.\int_{0}^{t} \frac{\partial \phi_{i B}}{\partial z}\right|_{z=h} d t,(i=1,2)
\end{aligned}
$$

in which, $f_{i A}$ denotes the sloshing height of the container potential function. $f_{i B}$ denotes the sloshing height of the liquid perturbed function. According to Eqs (12)-(26), one can take the container potential function to be

$$
\phi_{i A}=\dot{x}(t) r \cos \theta,(i=1,2,3,4)
$$

Introducing Eqs (25) and (27) into Eq. (22) gives

$$
\left.\frac{\partial \phi_{i B}}{\partial t}\right|_{z=h}+g f_{i B}=-\ddot{x}(t) r \cos \theta,(i=1,2)
$$

\section{Liquid perturbed functions}

It is well known that the mode superposition method is an effective approach to solve the dynamics response. However, this method requires a preliminary modal analysis. We have just developed an analytical method [31] to solve the natural frequencies and modes of the system considered here. By introducing the generalized coordinates $q_{n}(t)(n=1,2,3, \ldots)$, the liquid perturbed function $\phi_{i B}(r, \theta, z, t)$ can be written as

$$
\phi_{i B}=\cos \theta \sum_{n=1}^{\infty} \dot{q}_{n}(t) \Phi_{1 n}^{i}(r, z),(i=1,2,3,4)
$$

in which, $\Phi_{1 n}^{i}(r, z)$ is the natural mode shape of sub-domain $\Omega_{i}$ with the circumferential wave number equal to one. According to a recent paper [31], $\Phi_{1 n}^{i}(r, z)$ satisfies the following conditions: 


$$
\begin{aligned}
& \nabla^{2} \Phi_{1 n}^{i}=0, \quad(i=1,2,3,4) \\
& \left.\frac{\partial \Phi_{1 n}^{1}}{\partial z}\right|_{z=h_{2}}=0,\left.\frac{\partial \Phi_{1 n}^{1}}{\partial r}\right|_{r=R_{2}}=0, \\
& \left.\frac{\partial \Phi_{1 n}^{3}}{\partial z}\right|_{z=0}=0,\left.\frac{\partial \Phi_{1 n}^{3}}{\partial z}\right|_{z=h_{2}}=0,\left.\frac{\partial \Phi_{1 n}^{3}}{\partial r}\right|_{r=R_{2}}=0 \\
& \left.\frac{\partial \Phi_{1 n}^{4}}{\partial z}\right|_{z=0}=0 \\
& \left.\frac{\partial \Phi_{1 n}^{1}}{\partial z}\right|_{z=h}-\left.\omega_{1 n}^{2} \Phi_{1 n}^{1}\right|_{z=h}=0 \\
& \left.\frac{\partial \Phi_{1 n}^{2}}{\partial z}\right|_{z=h}-\left.\omega_{1 n}^{2} \Phi_{1 n}^{2}\right|_{z=h}=0 \\
& \Phi_{1 n}^{i}=\Phi_{1 n}^{l} \text { on } \Gamma_{k} \\
& \frac{\partial \Phi_{1 n}^{i}}{\partial \vec{\tau}_{k}}=\frac{\partial \Phi_{1 n}^{l}}{\partial \vec{\tau}_{k}} \text { on } \Gamma_{k} \\
& \frac{\partial \Phi_{1 n}^{i}}{\partial \vec{n}_{k}}=\frac{\partial \Phi_{1 n}^{l}}{\partial \vec{n}_{k}} \text { on } \Gamma_{k}
\end{aligned}
$$

The $n$th natural frequency $\omega_{1 n}$ and the corresponding natural mode $\Phi_{1 n}^{i}$ can be obtained by using the sub-domain method [31]. We further consider the $m$ th natural frequency $\omega_{1 m}\left(\omega_{1 n} \neq \omega_{1 m}\right)$ and the corresponding natural mode $\Phi_{1 m}^{i}$. According to the Gauss Theorem, one can obtained the following integral equations

$$
\begin{aligned}
& \iiint_{\Omega_{1}}\left[\nabla \Phi_{1 n}^{1} \cdot \nabla \Phi_{1 m}^{1}+\Phi_{1 m}^{1}\left(\nabla^{2} \Phi_{1 n}^{1}\right)\right] \mathrm{dV}=\oiint_{\partial \Omega_{1}} \Phi_{1 m}^{1} \nabla \Phi_{1 n}^{1} \cdot \mathrm{d} \overrightarrow{\mathrm{S}} \\
& \iiint_{\Omega_{2}}\left[\nabla \Phi_{1 n}^{2} \cdot \nabla \Phi_{1 m}^{2}+\Phi_{1 m}^{2}\left(\nabla^{2} \Phi_{1 n}^{2}\right)\right] \mathrm{dV}=\oiint_{\partial \Omega_{2}} \Phi_{1 m}^{2} \nabla \Phi_{1 n}^{2} \cdot \mathrm{d} \overrightarrow{\mathrm{S}} \\
& \iiint_{\Omega_{3}}\left[\nabla \Phi_{1 n}^{3} \cdot \nabla \Phi_{1 m}^{3}+\Phi_{1 m}^{3}\left(\nabla^{2} \Phi_{1 n}^{3}\right)\right] \mathrm{dV}=\oiint_{\partial \Omega_{3}} \Phi_{1 m}^{3} \nabla \Phi_{1 n}^{3} \cdot \mathrm{d} \overrightarrow{\mathrm{S}} \\
& \iiint_{\Omega_{4}}\left[\nabla \Phi_{1 n}^{4} \cdot \nabla \Phi_{1 m}^{4}+\Phi_{1 m}^{4}\left(\nabla^{2} \Phi_{1 n}^{4}\right)\right] \mathrm{dV}=\oiint_{\partial \Omega_{4}} \Phi_{1 m}^{4} \nabla \Phi_{1 n}^{4} \cdot \mathrm{d} \overrightarrow{\mathbf{S}}
\end{aligned}
$$

Adding Eqs (39)-(42) gives

$$
\sum_{i=1}^{4} \iiint_{\Omega_{i}}\left[\nabla \Phi_{1 n}^{i} \cdot \nabla \Phi_{1 m}^{i}+\Phi_{1 m}^{i}\left(\nabla^{2} \Phi_{1 n}^{i}\right)\right] \mathrm{dV}=\iint_{S_{1}} \Phi_{1 m}^{1} \nabla \Phi_{1 n}^{1} \cdot \mathrm{d} \overrightarrow{\mathrm{S}}+\iint_{S_{2}} \Phi_{1 m}^{2} \nabla \Phi_{1 n}^{2} \cdot \mathrm{d} \overrightarrow{\mathrm{S}}
$$

in which, $S_{1}$ denotes the free-surface of sub-domain $\Omega_{1}$. $S_{2}$ denotes the free-surface of sub-domain $\Omega_{2}$. Substituting Eq. (30) into Eq. (43) gives

$$
\sum_{i=1}^{4} \iiint_{\Omega_{i}} \nabla \Phi_{1 n}^{i} \cdot \nabla \Phi_{1 m}^{i} \mathrm{dV}=\iint_{S_{1}} \Phi_{1 m}^{1} \nabla \Phi_{1 n}^{1} \cdot \mathrm{d} \overrightarrow{\mathrm{S}}+\iint_{S_{2}} \Phi_{1 m}^{2} \nabla \Phi_{1 n}^{2} \cdot \mathrm{d} \overrightarrow{\mathrm{S}}
$$


One can obtain a similar integral equation by the same procedure

$$
\sum_{i=1}^{4} \iiint_{\Omega_{i}} \nabla \Phi_{1 m}^{i} \cdot \nabla \Phi_{1 n}^{i} \mathrm{dV}=\iint_{S_{1}} \Phi_{1 n}^{1} \nabla \Phi_{1 m}^{1} \cdot \mathrm{d} \overrightarrow{\mathrm{S}}+\iint_{S_{2}} \Phi_{1 n}^{2} \nabla \Phi_{1 m}^{2} \cdot \mathrm{d} \overrightarrow{\mathrm{S}}
$$

According to Eqs (44) and (45), one has

$$
\iint_{S_{1}} \Phi_{1 m}^{1} \nabla \Phi_{1 n}^{1} \cdot \mathrm{d} \overrightarrow{\mathrm{S}}+\iint_{S_{2}} \Phi_{1 m}^{2} \nabla \Phi_{1 n}^{2} \cdot \mathrm{d} \overrightarrow{\mathrm{S}}=\iint_{S_{1}} \Phi_{1 n}^{1} \nabla \Phi_{1 m}^{1} \cdot \mathrm{d} \overrightarrow{\mathrm{S}}+\iint_{S_{2}} \Phi_{1 n}^{2} \nabla \Phi_{1 m}^{2} \cdot \mathrm{d} \overrightarrow{\mathrm{S}}
$$

Substituting Eqs (34) and (35) into Eq. (46) obtains the following orthotropic conditions:

$$
\begin{aligned}
& \iint_{S_{1}} \Phi_{1 m}^{1} \Phi_{1 n}^{1} \vec{n} \cdot \mathrm{d} \overrightarrow{\mathrm{S}}+\iint_{S_{2}} \Phi_{1 m}^{2} \Phi_{1 n}^{2} \vec{n} \cdot \mathrm{d} \overrightarrow{\mathrm{S}}=0 \\
& \iint_{S_{1}} \Phi_{1 m}^{1} \nabla \Phi_{1 n}^{1} \cdot \mathrm{d} \overrightarrow{\mathrm{S}}+\iint_{S_{2}} \Phi_{1 m}^{2} \nabla \Phi_{1 n}^{2} \cdot \mathrm{d} \overrightarrow{\mathrm{S}}=0
\end{aligned}
$$

In order to simplify Eqs (47) and (48), we take $\Phi_{1 n}$ to be the natural mode of the liquid domain $\Omega$, namely,

$$
\Phi_{1 n}(r, z)=\Phi_{1 n}^{i}(r, z),(r, \theta, z) \in \Omega_{i},(i=1,2,3,4)
$$

Equations (47) and (48) can be rewritten as the following form:

$$
\begin{aligned}
& \iint_{S} \Phi_{1 m} \Phi_{1 n} \vec{n} \cdot \mathrm{d} \overrightarrow{\mathrm{S}}=0 \\
& \iint_{S} \Phi_{1 m} \nabla \Phi_{1 n} \cdot \mathrm{d} \overrightarrow{\mathrm{S}}=0
\end{aligned}
$$

\section{Equation of dynamic response}

Introducing Eqs (26) and (29) into Eq. (28) gives

$$
\left.\sum_{n=1}^{\infty} \ddot{q}_{n}(t) \Phi_{1 n}(r, z)\right|_{z=h}+\left.g \sum_{n=1}^{\infty} q_{n}(t) \frac{\partial \Phi_{1 n}(r, z)}{\partial z}\right|_{z=h}=-\ddot{x}(t) r
$$

Expanding the right side of Eq. (52) obtains

$$
\left.\sum_{n=1}^{\infty} \ddot{q}_{n}(t) \Phi_{1 n}(r, z)\right|_{z=h_{1}}+\left.g \sum_{n=1}^{\infty} q_{n}(t) \frac{\partial \Phi_{1 n}(r, z)}{\partial z}\right|_{z=h}=-\left.\ddot{x}(t) \sum_{n=1}^{\infty} b_{n} \Phi_{1 n}(r, z)\right|_{z=h}
$$

in which, $b_{n}(n=1,2,3, \ldots)$ is the expansion coefficient, which can be determined by

$$
b_{n}=\frac{\left.\int_{0}^{R_{2}} r^{2} \Phi_{1 n}(r, z)\right|_{z=h} d r}{\int_{0}^{R_{2}} r\left[\left.\Phi_{1 n}(r, z)\right|_{z=h}\right]^{2} d r}
$$

The space coordinate $r$ in Eq. (53) can be eliminated by multiplying two sides of equation by $\Phi_{1 n}(r, z)$ and making the integral of $r$ on the interval $\left[0, R_{2}\right]$. According to the orthotropic conditions given in Eqs (50) and (51), one can obtain the following equation for each generalized coordinate $q_{n}(t)$ :

$$
\begin{aligned}
& \ddot{q}_{n}(t) \int_{0}^{R_{2}} r\left[\left.\Phi_{1 n}(r, z)\right|_{z=h}\right]^{2} d r+\left.g q_{n}(t) \int_{0}^{R_{2}} r\left[\frac{\partial \Phi_{1 n}(r, z)}{\partial z} \Phi_{1 n}(r, z)\right]\right|_{z=h} d r= \\
& -\left.\ddot{x}(t) \int_{0}^{R_{2}} r^{2} \Phi_{1(n)}(r, z)\right|_{z=h} d r, \quad(n=1,2,3, \ldots)
\end{aligned}
$$


The generalized coordinate $q_{n}(t)$ can be obtained by solving the above second-order common differential equation. For harmonic lateral excitation, Eq. (55) can be solved analytically. For seismic lateral excitation, Eq. (55) can be solved numerically by the Newmark beta method. Substituting $q_{n}(t)$ back into Eq. (29) gives the liquid perturbed function. By adding the liquid perturbed function $\varphi_{i B}$ to the container potential function $\varphi_{i A}$, one obtain the total potential function:

$$
\phi_{i}=\cos \theta\left(\sum_{n=1}^{\infty} \dot{q}_{n}(t) \Phi_{1 n}^{i}(r, z)+\dot{x}(t) r\right),(i=1,2,3,4)
$$

Substituting Eq. (56) into Eq. (10) gives the sloshing surface displacement:

$$
f_{i}=-\frac{\cos \theta}{g}\left(\left.\sum_{n=1}^{\infty} \ddot{q}_{n}(t) \Phi_{1 n}^{i}(r, z)\right|_{z=h}+\ddot{x}(t) r\right),(i=1,2)
$$

The hydrodynamic pressure at any point due to liquid sloshing (neglecting the hydrostatic pressure, $\rho g z$ ) can be determined from the following pressure equation:

$$
P_{i}=-\rho \frac{\partial \varphi_{i}}{\partial t}=-\rho \cos \theta\left(\sum_{n=1}^{\infty} \ddot{q}_{n}(t) \Phi_{1 n}^{i}(r, z)+\ddot{x}(t) r\right), \quad(i=1,2,3,4)
$$

The resultant force acting on the container wall is obtained by integrating the pressure over the corresponding area of the boundary. Considering the excitation along $\theta=0$, one can obtain the resultant force along the $x$-axis

$$
F_{x}=\int_{0}^{2 \pi} \int_{h_{2}}^{h} P_{1}\left(R_{2}, \theta, z, t\right) \cos \theta R_{2} \mathrm{~d} z \mathrm{~d} \theta+\int_{0}^{2 \pi} \int_{0}^{h_{2}} P_{3}\left(R_{2}, \theta, z, t\right) \cos \theta R_{2} \mathrm{~d} z \mathrm{~d} \theta
$$

The hydrodynamic moment due to liquid pressure acting on the wall with the center of the bottom is

$$
M_{W}=\left.\int_{0}^{2 \pi} \int_{h_{2}}^{h} P_{1}(r, \theta, z, t)\right|_{r=R_{2}} z \cos \theta R_{2} \mathrm{~d} z \mathrm{~d} \theta+\left.\int_{0}^{2 \pi} \int_{0}^{h_{2}} P_{3}(r, \theta, z, t)\right|_{r=R_{2}} z \cos \theta R_{2} \mathrm{~d} z \mathrm{~d} \theta
$$

The hydrodynamic moment due to liquid pressure acting on the baffle with the center of the bottom is

$$
M_{\text {baffle }}=\left.\int_{R_{1}}^{R_{2}} \int_{0}^{2 \pi} P_{1}(r, \theta, z, t)\right|_{z=h_{2}} r^{2} \cos \theta \mathrm{d} \theta \mathrm{d} r-\left.\int_{R_{1}}^{R_{2}} \int_{0}^{2 \pi} P_{3}(r, \theta, z, t)\right|_{z=h_{2}} r^{2} \cos \theta \mathrm{d} \theta \mathrm{d} r
$$

The hydrodynamic moment due to liquid pressure acting on the bottom with the center of the bottom is

$$
M_{\text {bottom }}=\left.\int_{0}^{R_{1}} \int_{0}^{2 \pi} P_{4}(r, \theta, z, t)\right|_{z=0} r^{2} \cos \theta \mathrm{d} \theta \mathrm{d} r+\left.\int_{R_{1}}^{R_{2}} \int_{0}^{2 \pi} P_{3}(r, \theta, z, t)\right|_{z=0} r^{2} \cos \theta \mathrm{d} \theta \mathrm{d} r
$$

The total moment $M_{y}$ about the $y$-axis is the sum of Eqs (60)-(62):

$$
M_{y}=M_{W}+M_{\text {baffle }}+M_{\text {bottom }}
$$

\section{Response to harmonic lateral excitation}

Assume the system shown in Fig. 3 is subjected to a harmonic excitation $x(t)$ in the form of sine-wave having the amplitude $X_{0}$ and the circular frequency $\bar{\omega}$. Equation (55) can be rewritten in the following form:

$$
\begin{aligned}
& \ddot{q}_{n}(t) \int_{0}^{R_{2}} r\left[\left.\Phi_{1 n}(r, z)\right|_{z=h}\right]^{2} d r+\left.g q_{n}(t) \int_{0}^{R_{2}} r\left[\frac{\partial \Phi_{1 n}(r, z)}{\partial z} \Phi_{1(n)}(r, z)\right]\right|_{z=h} d r= \\
& \left.\quad X_{0} \bar{\omega}^{2} \sin \bar{\omega} t \int_{0}^{R_{2}} r^{2} \Phi_{1 n}(r, z)\right|_{z=h} d r
\end{aligned}
$$

Before considering this case, one can have 
Table 1

Maximum slosh displacements $(m)$ at the container wall

$$
\begin{aligned}
M_{1 n} & =\frac{\int_{0}^{R_{2}} r\left[\left.\Phi_{1 n}(r, z)\right|_{z=h}\right]^{2} d r}{\left.\int_{0}^{R_{2}} r^{2} \Phi_{1 n}(r, z)\right|_{z=h} d r} \\
K_{1 n} & =\frac{\left.g \int_{0}^{R_{2}} r\left\{\left[\partial \Phi_{1 n}(r, z) / \partial z\right] \Phi_{1 n}(r, z)\right\}\right|_{z=h} d r}{\left.\int_{0}^{R_{2}} r^{2} \Phi_{1 n}(r, z)\right|_{z=h} d r}
\end{aligned}
$$

\begin{tabular}{|c|c|c|c|c|c|c|}
\hline \multirow[t]{2}{*}{$h_{1} / R_{2}$} & \multicolumn{2}{|c|}{$R_{1} / R_{2}=0.4$} & \multicolumn{2}{|c|}{$R_{1} / R_{2}=0.6$} & \multicolumn{2}{|c|}{$R_{1} / R_{2}=0.8$} \\
\hline & Present & Biswal [27] & Present & Biswal [27] & Present & Biswal [27] \\
\hline 0.1 & 0.0015 & 0.0016 & 0.0034 & 0.0036 & 0.0158 & 0.0166 \\
\hline 0.3 & 0.0049 & 0.0051 & 0.0095 & 0.01 & 0.033 & 0.0344 \\
\hline 0.8 & 0.0386 & 0.039 & 0.0405 & 0.0406 & 0.0412 & 0.0411 \\
\hline
\end{tabular}

in which, $M_{1 n}$ is the mass coefficient. $K_{1 n}$ is the stiffness coefficient. Introducing Eqs (65) and (66) into Eq. (64) gives

$$
\ddot{q}_{n}(t) M_{1 n}+q_{n}(t) K_{1 n}=X_{0} \bar{\omega}^{2} \sin \bar{\omega} t
$$

According to Eqs (34) and (35), one has $\omega_{1 n}^{2}=K_{1 n} / M_{1 n}$. For the system starting from rest, i.e., $q_{n}(0)=\dot{q}_{n}(0)=0$, the solution of Eq. (64) is

$$
q_{n}(t)=\frac{X_{0} \bar{\omega}^{2}}{K_{1 n}}\left(\frac{\omega_{1 n}^{2}}{\omega_{1 n}^{2}-\bar{\omega}^{2}}\right)\left(\sin \bar{\omega} t-\frac{\bar{\omega}}{\omega_{1 n}} \sin \omega_{1 n} t\right)
$$

in which, $\sin \bar{\omega} t$ represents the response component under the frequency of the applied excitation, it is called the steady-state response and is directly related to the excitation. Since in a practical case, the damping of liquid will cause the last term to vanish eventually, it is termed the transient response. It should be mentioned that when the excitation frequency $\bar{\omega}$ approaches a sloshing natural frequency $\omega_{1 n}, q_{n}(t)$ would close to infinity (i.e., resonance) because the damping effect is not considered in the present analysis. Therefore, if the excitation frequency is near the sloshing natural frequencies, the present solutions are only suitable for the qualitative analysis.

The sloshing displacement, the hydrodynamic pressure on the container wall, the resultant force along $x$-axis and hydrodynamic moment about $y$-axis with the center of the bottom will be discussed in Sections 6.1-6.4. In the following study, the radius of the container is fixed at $R_{2}=0.508 \mathrm{~m}$ and the liquid height is fixed at $h=0.508 \mathrm{~m}$. The excitation circular frequency is fixed at $\bar{\omega}=5.811 \mathrm{rad} / \mathrm{s}$ and the movement amplitude of the container is fixed at $X_{0}=0.001 \mathrm{~m}$. The density of the liquid is fixed at $1000 \mathrm{~kg} / \mathrm{m}^{3}$.

\subsection{Sloshing displacement}

Substituting Eq. (68) into Eq. (57) gives

$$
\begin{gathered}
f_{i}=-\frac{X_{0} \bar{\omega}^{3} \cos \theta}{g} \sum_{n=1}^{\infty} \frac{\Phi_{1 n}^{i}(r, h)}{K_{1 n}}\left(\frac{\omega_{1 n}^{2}}{\omega_{1 n}^{2}-\bar{\omega}^{2}}\right)\left(\omega_{1 n} \sin \omega_{1 n} t-\bar{\omega} \sin \bar{\omega} t\right)+ \\
\frac{X_{0} \bar{\omega}^{2}}{g} \sin (\bar{\omega} t) r \cos \theta,(i=1,2)
\end{gathered}
$$

The maximum sloshing displacement at the container wall is computed for the parameters: $R_{1} / R_{2}=0.4,0.6,0.8$ and $h_{1} / R_{2}=0.1,0.3,0.8$. Biswal [27] studied the same problem by the finite element method. The comparison study of the results is given in Table 1. Good agreements between the present results and the Biswal's results are observed. The sloshing response of liquid in the cylindrical rigid container with the rigid baffle is studied under the loading condition within the first $10 \mathrm{~s}$. The sloshing displacement $f_{\text {wall }}$ at the container wall for $h_{1} / R_{2}=0.3$, $R_{1} / R_{2}=0.4,0.6,0.8$ is given in Fig. 4. The sloshing displacement $f_{\text {wall }}$ at the container wall for $R_{1} / R_{2}=0.6$, $h_{1} / R_{2}=0.1,0.3,0.8$ is given in Fig. 5. It can be seen from Figs 4 and 5 that the sloshing displacement at the container wall decreases with the decrease of $R_{1} / R_{2}$ and $h_{1} / R_{2}$. Namely, small $R_{1} / R_{2}$ and $h_{1} / R_{2}$, such as $R_{1} / R_{2} \leqslant 0.6$ and $h_{1} / R_{2} \leqslant 0.6$, can remarkably decrease the sloshing amplitude of liquid. 


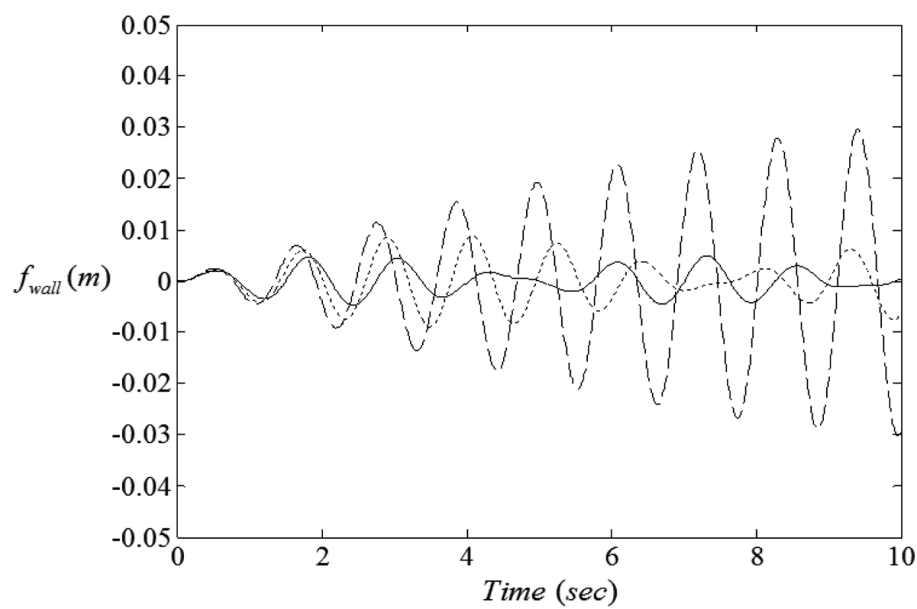

Fig. 4. Sloshing displacement at the container wall under the harmonic excitation for $h_{1} / R_{2}=0.3$ and three different $R_{1} / R_{2}\left(-R_{1} / R_{2}=\right.$ $\left.0.4, \cdots \cdots R_{1} / R_{2}=0.6,---R_{1} / R_{2}=0.8\right)$.

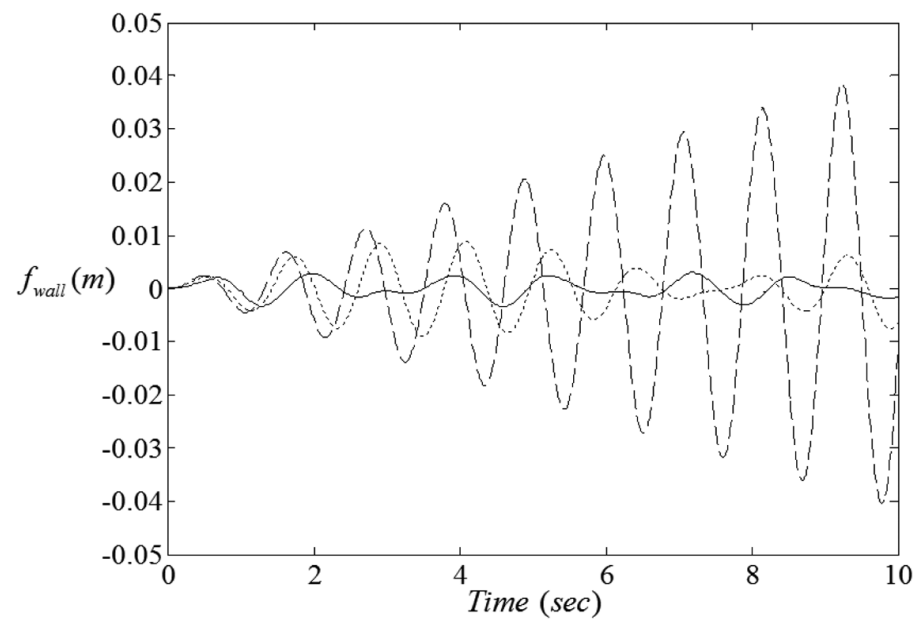

Fig. 5. Sloshing displacement at the container wall under the harmonic excitation for $R_{1} / R_{2}=0.6$ and three different $h_{1} / R_{2}\left(-h_{1} / R_{2}=\right.$ $\left.0.1, \cdots \cdots h_{1} / R_{2}=0.3,---h_{1} / R_{2}=0.8\right)$

\subsection{Hydrodynamic pressure}

Substituting Eq. (68) into Eq. (58) gives

$$
\begin{gathered}
P_{i}=-\rho \frac{\partial \varphi_{i}}{\partial t}=-\rho X_{0} \bar{\omega}^{3} \cos \theta \sum_{n=1}^{\infty} \frac{\Phi_{1 n}^{i}(r, z)}{K_{1 n}}\left(\frac{\omega_{1 n}^{2}}{\omega_{1 n}^{2}-\bar{\omega}^{2}}\right)\left(\omega_{1 n} \sin \omega_{1 n} t-\bar{\omega} \sin \bar{\omega} t\right)+ \\
\rho X_{0} \bar{\omega}^{2} \sin (\bar{\omega} t) r \cos \theta,(i=1,2,3,4)
\end{gathered}
$$

Since in a practical case, the damping of liquid will cause the transient response to vanish eventually. The analysis focused on the steady-state response is more significant than the transient response. According to Eq. (70), one can obtain the component of hydrodynamic pressure which is related to the steady-state response:

$$
P_{i s t}=\rho X_{0} \bar{\omega}^{4} \cos \theta \sin \bar{\omega} t \sum_{n=1}^{\infty} \frac{\Phi_{1 n}^{i}(r, z)}{K_{1 n}}\left(\frac{\omega_{1 n}^{2}}{\omega_{1 n}^{2}-\bar{\omega}^{2}}\right)+\rho X_{0} \bar{\omega}^{2} \sin (\bar{\omega} t) r \cos \theta,(i=1,2,3,4)
$$




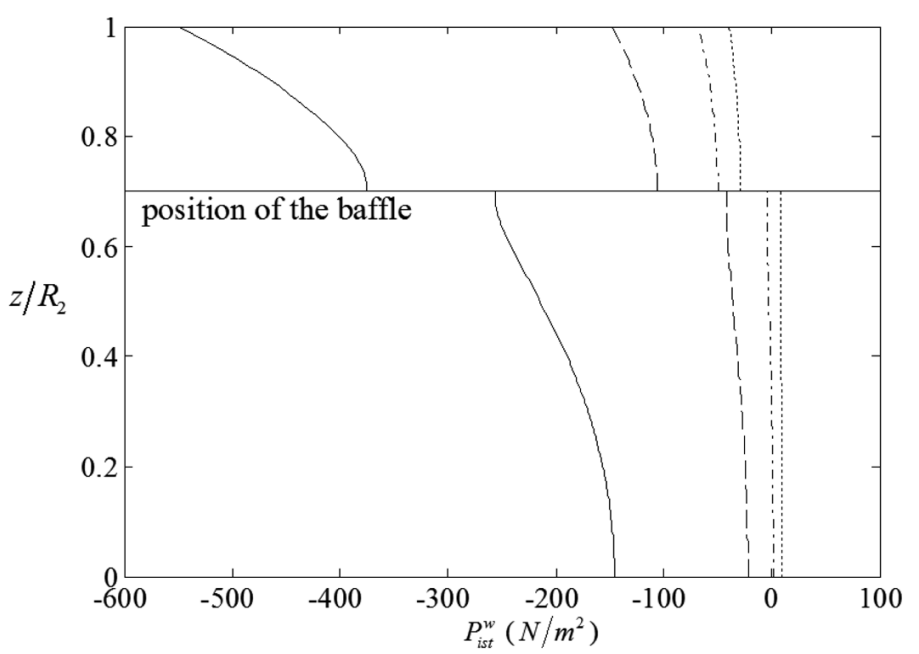

Fig. 6. The wall pressure distribution $P_{i s t}^{w}$ at $\theta=0, \bar{\omega} t=\pi / 2$ for $h_{1} / R_{2}=0.3$, and four different $R_{1} / R_{2}\left(-R_{1} / R_{2}=0.9\right.$, --$\left.R_{1} / R_{2}=0.8,-\cdot-R_{1} / R_{2}=0.7, \cdots \cdots R_{1} / R_{2}=0.6\right)$.

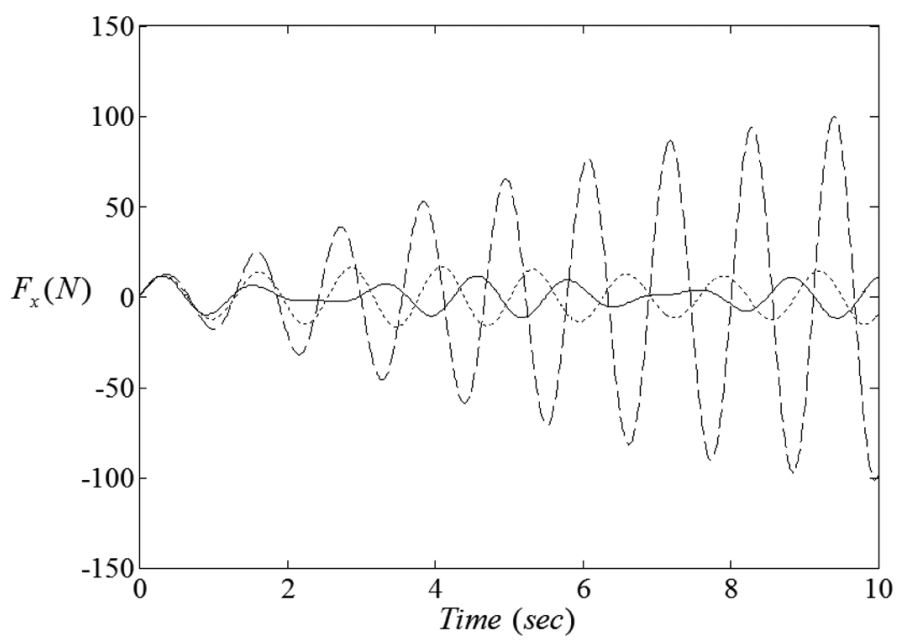

Fig. 7. The resultant hydrodynamic force under the harmonic excitation for $h_{1} / R_{2}=0.3$ and three different $R_{1} / R_{2}\left(-R_{1} / R_{2}=0.4, \cdots \cdots\right.$ $\left.R_{1} / R_{2}=0.6,--R_{1} / R_{2}=0.8\right)$.

According to Eq. (71), one can conclude that the maximum pressure occurs on the wall at $\theta=0, \bar{\omega} t=\pi / 2$. It is given by the expression:

$$
P_{i s t}^{w}=\rho X_{0} \bar{\omega}^{4} \sum_{n=1}^{\infty} \frac{\Phi_{1 n}^{i}\left(R_{2}, z\right)}{K_{1 n}}\left(\frac{\omega_{1 n}^{2}}{\omega_{1 n}^{2}-\bar{\omega}^{2}}\right)+\rho X_{0} \bar{\omega}^{2} R_{2},(i=1,3)
$$

The curves in Fig. 6 indicate the maximum pressure distribution $P_{i s t}^{w}$ on the wall for the parameters $h_{1} / R_{2}=0.3$, $R_{1} / R_{2}=0.6,0.7,0.8,0.9$, respectively. It is seen from Fig. 6 that the continuity of the hydrodynamic pressure along the wall are cut off by the baffle. Namely, the pressure distribution is divided into two parts by the baffle. The liquid part above the baffle behaves like a sloshing part. The liquid part below the baffle behaves like a rigid part. It is seen from Fig. 6 that the liquid the hydrodynamic pressure on the wall above the baffle is significantly larger than that below the baffle. Moreover, it is seen from Fig. 6 that the hydrodynamic pressure on the container wall remarkably decreases with the decrease of $R_{1} / R_{2}$. 


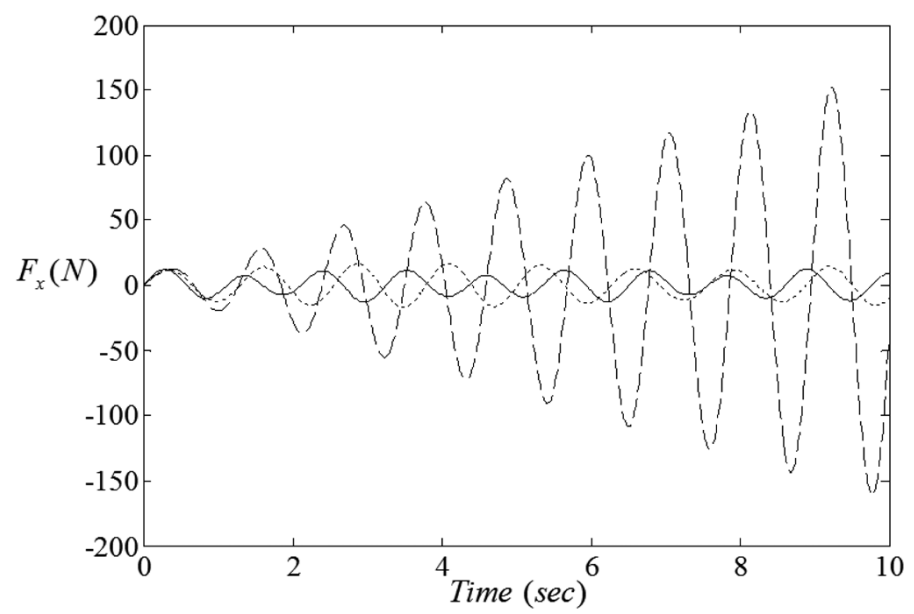

Fig. 8. The resultant hydrodynamic force under the harmonic excitation for $R_{1} / R_{2}=0.6$ and three different $h_{1} / R_{2}\left(-h_{1} / R_{2}=0.1, \cdots\right.$ $\left.h_{1} / R_{2}=0.3,---h_{1} / R_{2}=0.8\right)$.

\subsection{Resultant force}

Substituting Eq. (70) into Eqs (59) gives

$$
\begin{aligned}
& F_{x}=-\rho X_{0} \bar{\omega}^{3} \pi R_{2} \sum_{n=1}^{\infty} \frac{\int_{0}^{h} \Phi_{1 n}\left(R_{2}, z\right) \mathrm{d} z}{K_{1 n}}\left(\frac{\omega_{1 n}^{2}}{\omega_{1 n}^{2}-\bar{\omega}^{2}}\right)\left(\omega_{1 n} \sin \omega_{1 n} t-\bar{\omega} \sin \bar{\omega} t\right)+ \\
& \rho \pi X_{0} \bar{\omega}^{2} \sin (\bar{\omega} t) R_{2}^{2} h
\end{aligned}
$$

The resultant hydrodynamic force of liquid sloshing in the cylindrical rigid container with the rigid baffle is studied under the loading condition within the first $10 \mathrm{~s}$. The resultant force for $h_{1} / R_{2}=0.3, R_{1} / R_{2}=0.4,0.6,0.8$ is given in Fig. 7. The resultant force for $R_{1} / R_{2}=0.6, h_{1} / R_{2}=0.1,0.3,0.8$ is given in Fig. 8. It is seen from Figs 7 and 8 that the resultant hydrodynamic force remarkably decreases with the decrease of $h_{1} / R_{2}$ and $R_{1} / R_{2}$.

\subsection{Resultant moment}

Substituting Eq. (70) into Eqs (60)-(62) gives

$$
\begin{gathered}
M_{W}=-\rho \pi R_{2} \bar{\omega}^{2}\left[X_{0} \bar{\omega} \sum_{n=1}^{\infty} \frac{1}{K_{1 n}}\left(\frac{\omega_{1 n}^{2}}{\omega_{1 n}^{2}-\bar{\omega}^{2}}\right)\left(\omega_{1 n} \sin \omega_{1 n} t-\bar{\omega} \sin \bar{\omega} t\right) \times\right. \\
\left.\int_{0}^{h} z \Phi_{1 n}\left(R_{2}, z\right) \mathrm{d} z-\frac{X_{0} \sin (\bar{\omega} t) R_{2} h^{2}}{2}\right] \\
M_{\text {baffle }}=-\rho \pi X_{0} \bar{\omega}^{3} \sum_{n=1}^{\infty} \frac{1}{K_{1 n}}\left(\frac{\omega_{1 n}^{2}}{\omega_{1 n}^{2}-\bar{\omega}^{2}}\right)\left(\omega_{1 n} \sin \omega_{1 n} t-\bar{\omega} \sin \bar{\omega} t\right) \times \\
\left(\int_{R_{1}}^{R_{2}} \Phi_{1 n}^{1}\left(r, h_{2}\right) r^{2} \mathrm{~d} r-\int_{R_{1}}^{R_{2}} \Phi_{1 n}^{3}\left(r, h_{2}\right) r^{2} \mathrm{~d} r\right) \\
M_{b o t t o m}=-\rho \pi \bar{\omega}^{2}\left[X_{0} \bar{\omega} \sum_{n=1}^{\infty} \frac{1}{K_{1 n}}\left(\frac{\omega_{1 n}^{2}}{\omega_{1 n}^{2}-\bar{\omega}^{2}}\right)\left(\omega_{1 n} \sin \omega_{1 n} t-\bar{\omega} \sin \bar{\omega} t\right) \times\right. \\
\left.\int_{0}^{R_{2}} \Phi_{1 n}(r, 0) r^{2} \mathrm{~d} r-\frac{X_{0} \sin (\bar{\omega} t) R_{2}^{4}}{4}\right]
\end{gathered}
$$




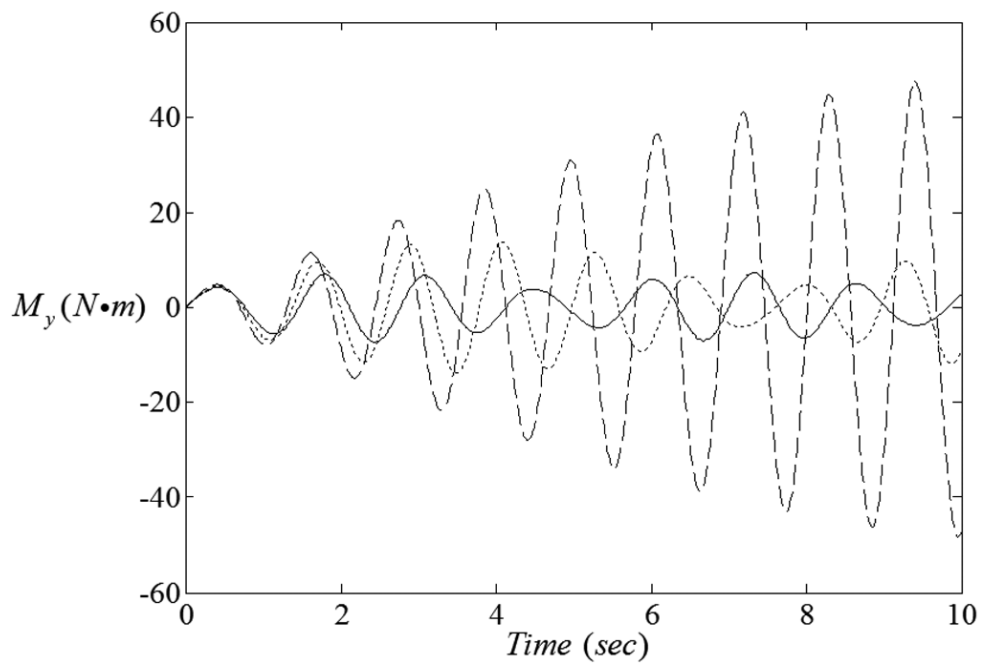

Fig. 9. The resultant hydrodynamic moment with the center of the bottom under the harmonic excitation for $h_{1} / R_{2}=0.3$ and three different $R_{1} / R_{2}\left(-R_{1} / R_{2}=0.4, \cdots \cdots R_{1} / R_{2}=0.6,---R_{1} / R_{2}=0.8\right)$.

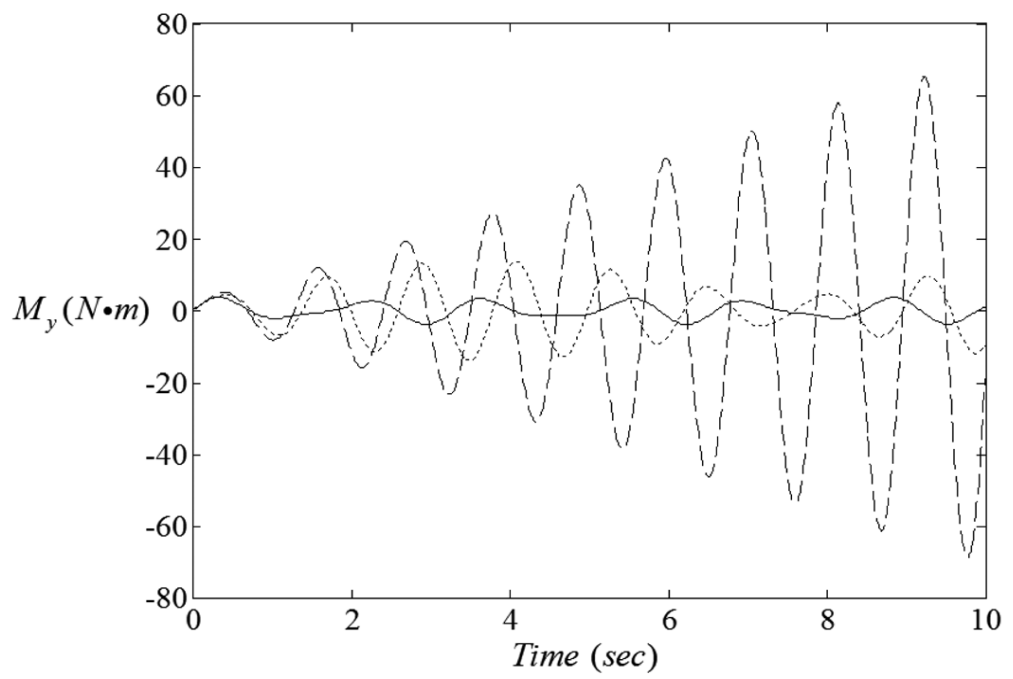

Fig. 10. The resultant hydrodynamic moment with the center of the bottom under the harmonic excitation for $R_{1} / R_{2}=0.6$ and three different $h_{1} / R_{2}\left(-h_{1} / R_{2}=0.1, \cdots \cdots h_{1} / R_{2}=0.3,---h_{1} / R_{2}=0.8\right)$.

The hydrodynamic moment response with the center of the bottom in the cylindrical rigid container with the rigid baffle is studied under the loading condition within the first $10 \mathrm{~s}$. The hydrodynamic moment $M_{y}$ for $h_{1} / R_{2}=0.3$, $R_{1} / R_{2}=0.4,0.6,0.8$ is given in Fig. 9. The hydrodynamic moment $M_{y}$ for $R_{1} / R_{2}=0.6, h_{1} / R_{2}=0.1,0.3,0.8$ is given in Fig. 10. It is seen from Figs 9 and 10 that small $R_{1} / R_{2}$ and $h_{1} / R_{2}$, such as $R_{1} / R_{2} \leqslant 0.6$ and $h_{1} / R_{2} \leqslant 0.6$, can remarkably decrease the resultant hydrodynamic moment of liquid with the center of the bottom.

\section{Response to seismic lateral excitation}

The Newmark beta method is a general approach to dynamic response analysis, and it is well suited to solve Eq. (55) when the system is subjected to a seismic loading. The seismic record with $0.02 s$ interval, which is used in the following numerical examples, belongs to the north-south component recorded at Kobe Japanese Meteorological 


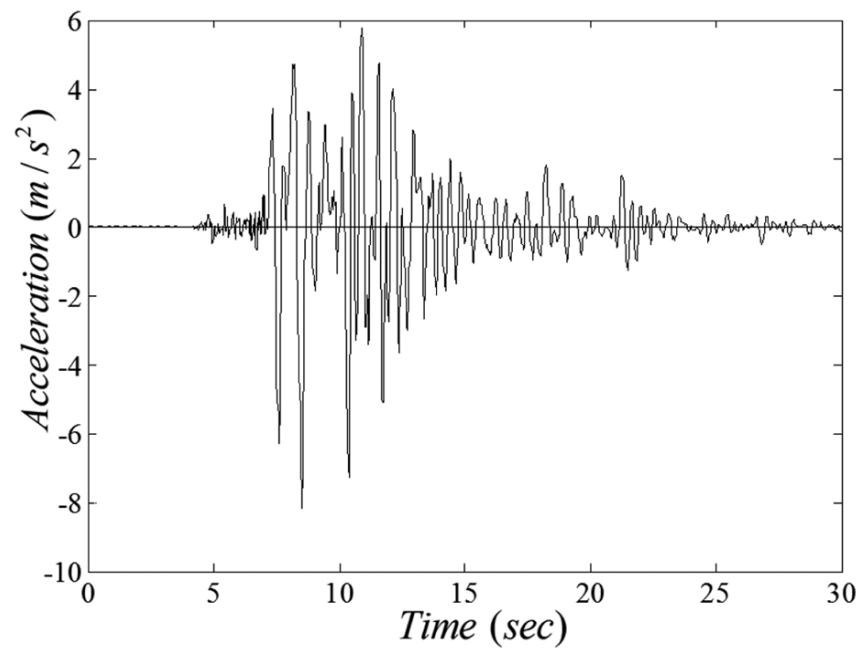

Fig. 11. Kobe seismic records within the first 30s.

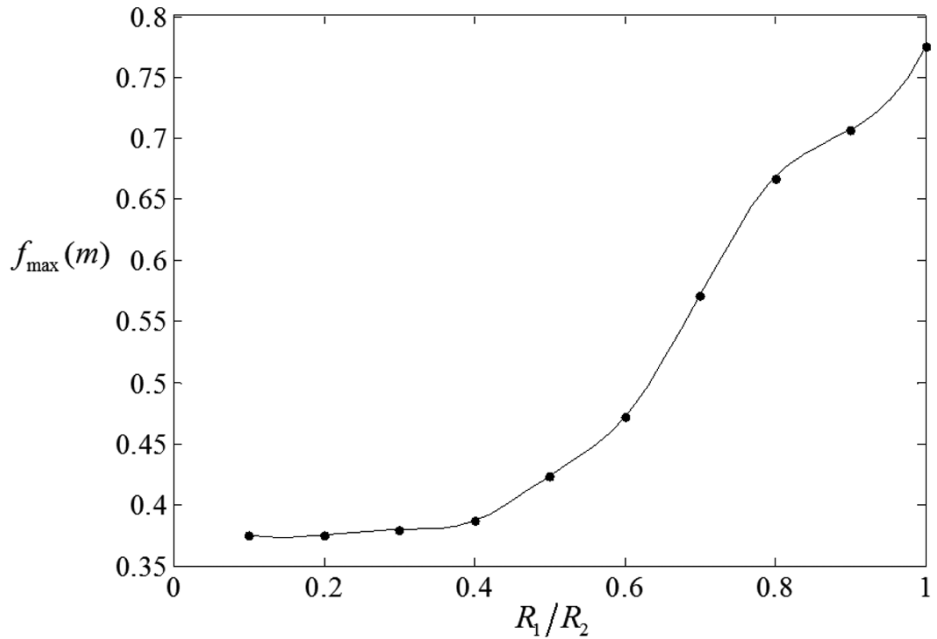

Fig. 12. Maximum amplitude in the time history of the sloshing displacement at the container wall under the Kobe seismic excitation versus $R_{1} / R_{2}$ for $h_{1} / R_{2}=0.4$.

Agency station during the 1995 Hyogo-ken Nanbu (Kobe) earthquake in Japan. A 30s seismic record is shown in Fig. 11. The sloshing response of liquid in the cylindrical rigid container with a rigid baffle is studied under the seismic lateral excitation. We consider the radius of the container $R_{2}=10 \mathrm{~m}$ and the liquid height $h=10 \mathrm{~m}$. The density of the liquid is $1000 \mathrm{~kg} / \mathrm{m}^{3}$. The sloshing displacement at the container wall, the resultant hydrodynamic force and the resultant hydrodynamic moment with the center of the bottom are studied for parameters: $h_{1} / R_{2}=$ $0.4, R_{1} / R_{2}$ from 0.1 to 1 and $R_{1} / R_{2}=0.4, h_{1} / R_{2}$ from 0.1 to 1 . The maximum amplitude in the time history of the sloshing displacement at the container wall versus $R_{1} / R_{2}$ for $h_{1} / R_{2}=0.4$ are illustrated in Fig. 12. The maximum amplitude in the time history of the sloshing displacement at the container wall versus $h_{1} / R_{2}$ for $R_{1} / R_{2}=0.4$ are illustrated in Fig. 13. It is seen from Figs 12 and 13 that the amplitude of the sloshing displacement at the container wall decreases with the decrease of $R_{1} / R_{2}$ and $h_{1} / R_{2}$. The maximum amplitude in the time history of the resultant hydrodynamic force versus $R_{1} / R_{2}$ for $h_{1} / R_{2}=0.4$ are illustrated in Fig. 14. The maximum amplitude in the time history of the resultant hydrodynamic force versus $h_{1} / R_{2}$ for $R_{1} / R_{2}=0.4$ are illustrated in Fig. 15 . From Figs 14 and 15, one can see that the amplitude of the resultant hydrodynamic force decreases with the increase of $h_{1} / R_{2}$ and $R_{1} / R_{2}$. The maximum amplitude in the time history of the resultant hydrodynamic moment with the center of 


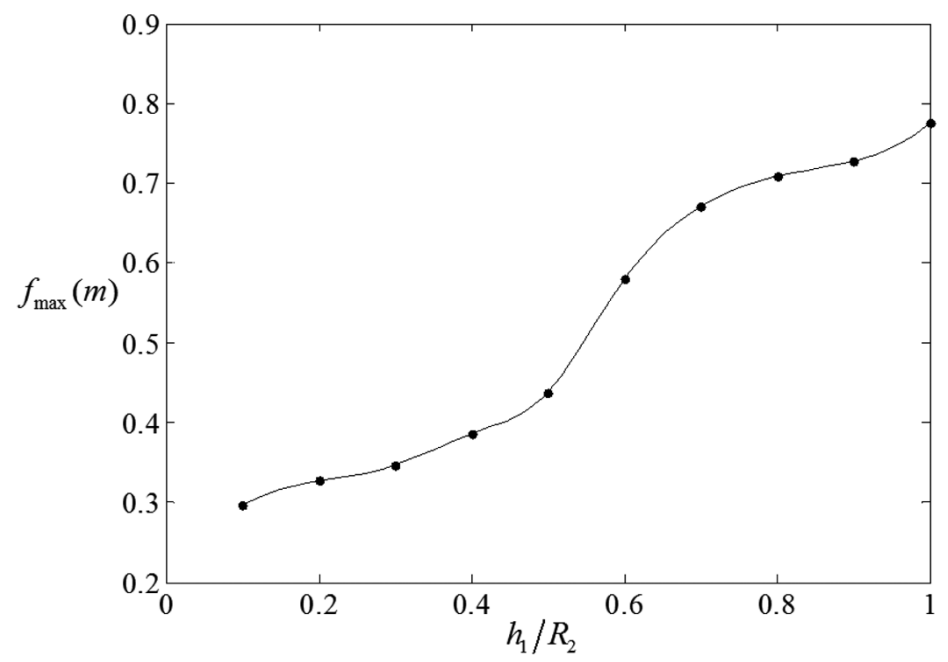

Fig. 13. Maximum amplitude in the time history of the sloshing displacement at the container wall under the Kobe seismic excitation versus $h_{1} / R_{2}$ for $R_{1} / R_{2}=0.4$.

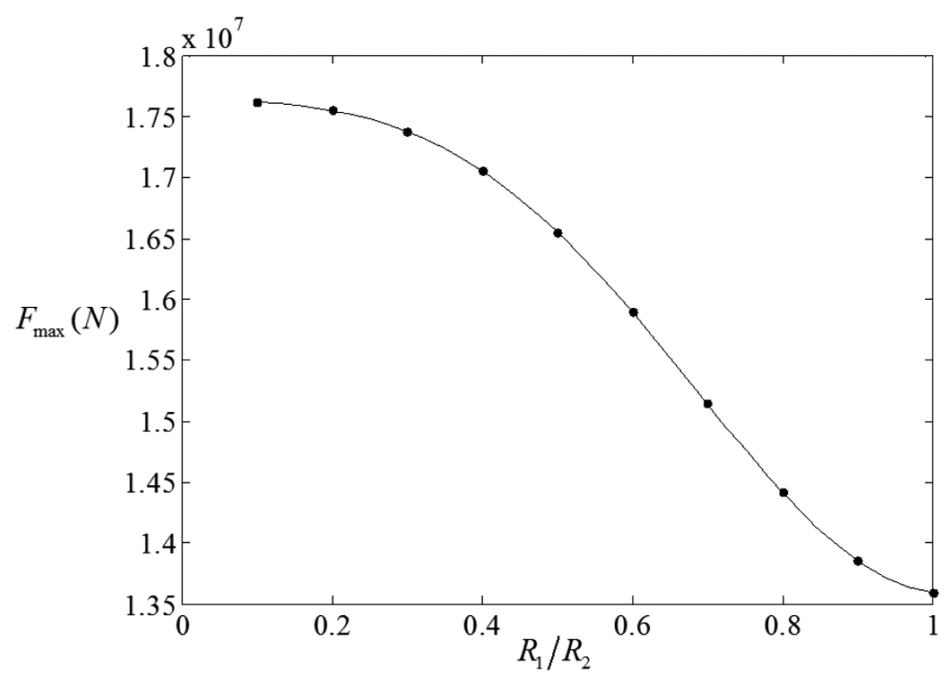

Fig. 14. Maximum amplitude in the time history of the resultant hydrodynamic force under the Kobe seismic excitation versus $R_{1} / R_{2}$ for $h_{1} / R_{2}=0.4$.

the bottom versus $R_{1} / R_{2}$ for $h_{1} / R_{2}=0.4$ are illustrated in Fig. 16. The maximum amplitude in the time history of the resultant hydrodynamic moment with the center of the bottom versus $h_{1} / R_{2}$ for $R_{1} / R_{2}=0.4$ are illustrated in Fig. 17. One can see from Fig. 16 that the maximum amplitude in the time history of the resultant hydrodynamic moment decreases with the decrease of $R_{1} / R_{2}$. Moreover, it is seen from Fig. 17 that the maximum amplitude in the time history of the resultant hydrodynamic moment decreases with the increase of $h_{1} / R_{2}$ when $h_{1} / R_{2}<0.42$, however, increases with the increase of $h_{1} / R_{2}$ when $h_{1} / R_{2}>0.42$.

\section{Conclusions}

Based on the modal analysis in advance, a mode superposition method is developed to study the sloshing response of liquid in a rigid cylindrical container with a rigid annual baffle under harmonic lateral loads and seismic lateral 


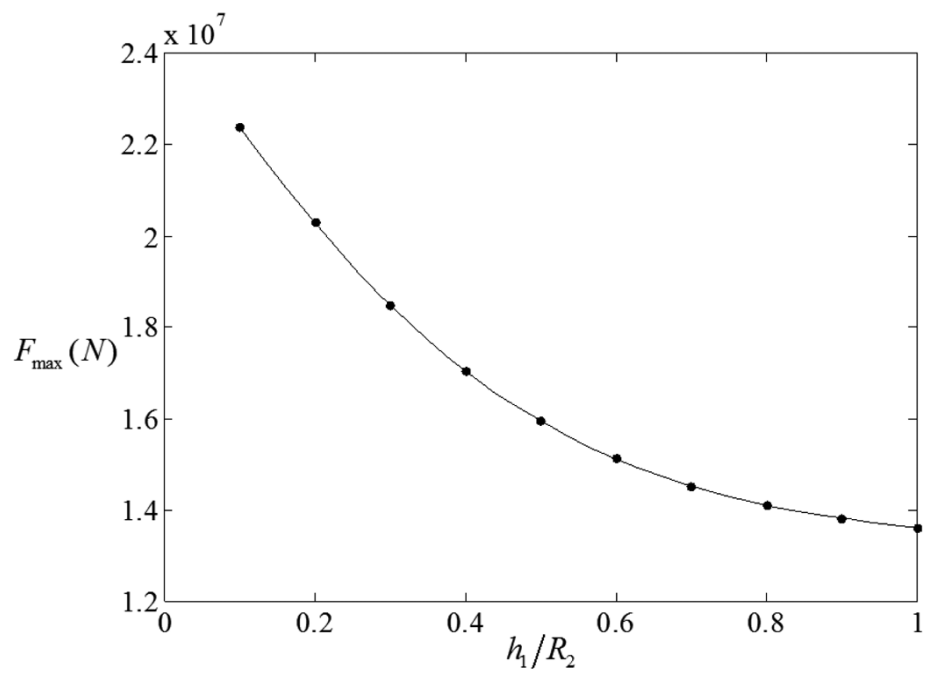

Fig. 15. Maximum amplitude in the time history of the resultant hydrodynamic force under the Kobe seismic excitation versus $h_{1} / R_{2}$ for $R_{1} / R_{2}=0.4$.

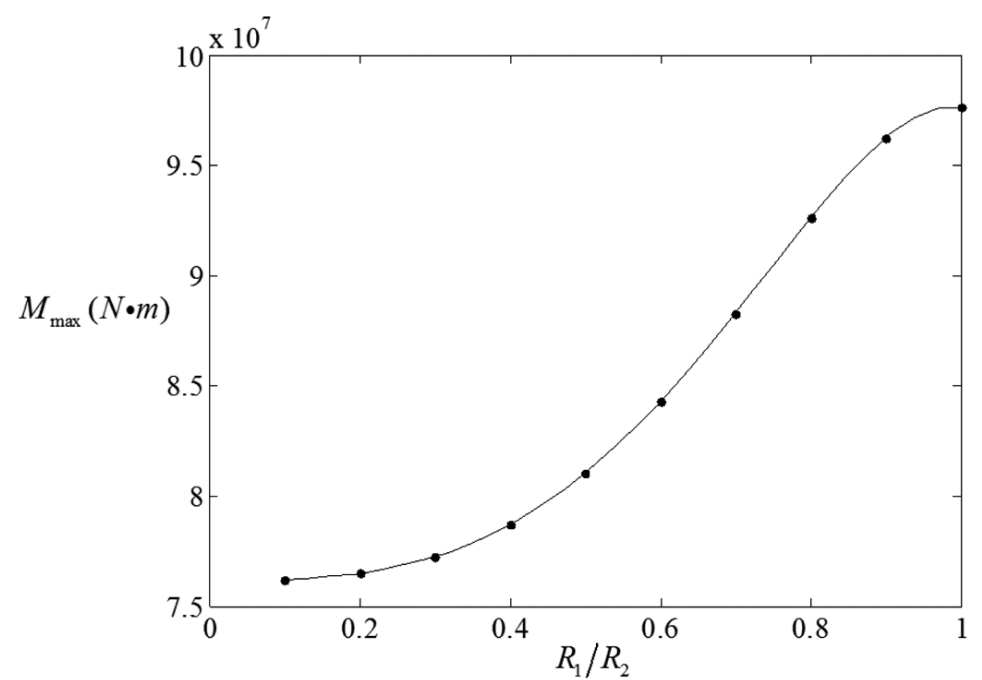

Fig. 16. Maximum amplitude in the time history of the resultant hydrodynamic moment with the center of the bottom of liquid under the Kobe seismic excitation versus $R_{1} / R_{2}$ for $h_{1} / R_{2}=0.4$.

loads. The rigid baffle with different radius ratios $R_{1} / R_{2}$ and different position ratios $h_{1} / R_{2}$ in a liquid-filled cylindrical rigid container have been checked. The following conclusions are obtained:

1. The baffle has an important effect on the dynamic properties of liquid in a container. The more the baffle closes to the free surface and the smaller the inner radius of the baffle, the more significant the effect of the baffle is.

2. Baffle destroys the continuity of the pressure distribution on the wall along the vertical direction. Namely, the behavior of the liquid can be divided into two parts. The liquid above the baffle behaves like a sloshing one. The liquid below the baffle behaves like a rigid one.

3. For the seismic excitation, the baffle with small inner radius and/or the baffle near to the free liquid surface will decrease the sloshing displacement, however, increase the resultant hydrodynamic force on the wall. The hydrodynamic moment decreases with the decrease of $R_{1} / R_{2}$ and $h_{1} / R_{2}$ when $h_{1} / R_{2}<0.42$, however, increases with the increase of $h_{1} / R_{2}$ when $h_{1} / R_{2}>0.42$. 


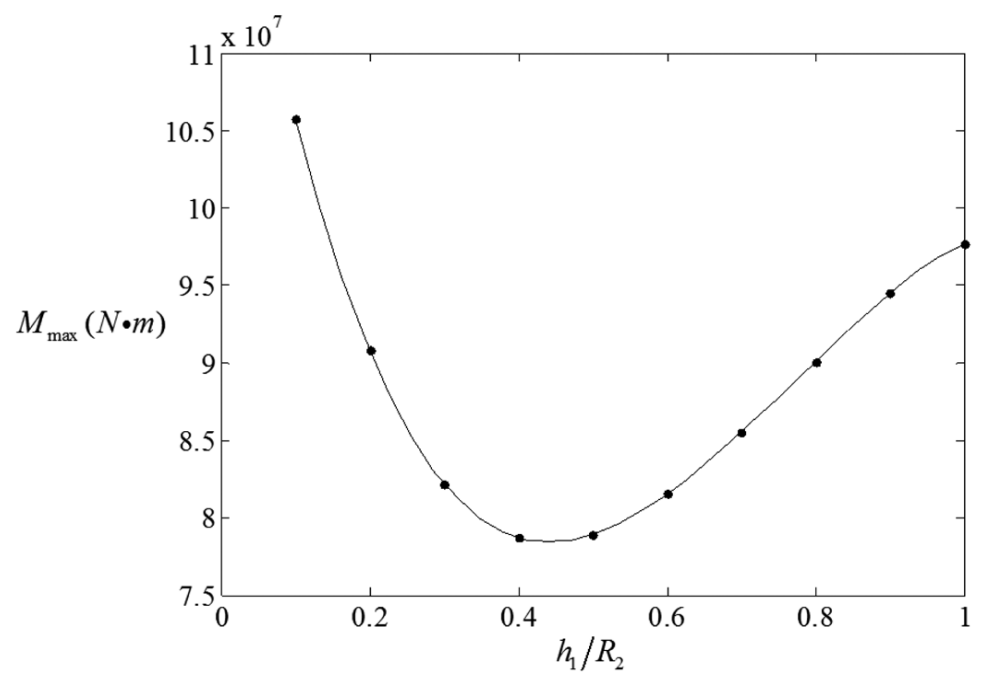

Fig. 17. Maximum amplitude in the time history of the resultant hydrodynamic moment with the center of the bottom of liquid under the Kobe seismic excitation versus $h_{1} / R_{2}$ for $R_{1} / R_{2}=0.4$.

\section{Acknowledgement}

The financial support from National Natural Science Foundation of China, Grant No. 11172123 is greatly acknowledged.

\section{References}

[1] R.A. Ibrahim, Liquid Sloshing Dynamics, Cambridge University Press, Cambridge, 2005.

[2] B.T. Morris, A laboratory model study of the behavior of liquid filled cylindrical tanks in earthquakes, Ph.D. Thesis, Stanford University, California, 1938

[3] P.W. Werner and K.J. Sundquist, On hydrodynamic earthquake effects, Transactions of American Geophysical Union 30(5) (1949), $636-657$.

[4] L.S. Jacobsen, Impulsive hydrodynamics of fluid inside a cylindrical container, Bulletin of the Seismological Society of America 39 (1949), 189-202.

[5] L.S. Jacobsen and R.S. Ayre, Hydrodynamic experiments with rigid cylindrical tanks subjected to transient motions, Bulletin of the Seismological Society of America 41(4) (1951), 313-346.

[6] L.M. Hoskins and L.S. Jacobsen, Water pressure in a tank caused by a simulated earthquake, Bulletin of the Seismological Society of America 47(1) (1957), 1-32.

[7] H.F. Bauer, The response of propellant in an arbitrary cylindrical tank due to single pulse excitation, Developments in Theoretical and Applied Mechanics 2, Edited by W.A. Shaw (1965), 351-383.

[8] K. Sogabe and H. Shibata, Response analysis on sloshing of liquid in a cylindrical storage Part 1: basic equation and response to sinusoidal input, Journal of Institute of Industrial Science 26(3) (1974), 119-122.

[9] K. Sogabe and H. Shibata, Response analysis on sloshing of liquid in a cylindrical storage Part 2: transient response to sinusoidal input, Journal of Institute of Industrial Science 26(4) (1974), 152-155.

[10] G.W. Housner, Dynamic pressures on accelerated fluid containers, Bulletin of the Seismological Society of America 47(1) (1957), 15-35.

[11] G.W. Housner, The dynamic behavior of water tanks, Bulletin of the Seismological Society of America 53(2) (1963), 381-387.

[12] M.A. Haroun and G.W. Housner, Seismic design of liquid storage tanks, Journal of the Technical Councils of ASCE 107(1) (1981), 191-207.

[13] J.S. Meserole and A. Fortini, Slosh dynamics in a toroidal tank, Journal of Spacecraft and Rockets 24(6) (1987), 523-531.

[14] H.N. Abramson and G.E. Ransleben, Some comparisons of sloshing behavior in cylindrical tanks with flat and conical bottoms, ARS Journal (1961), 542-544.

[15] H.N. Abramson, The dynamic behavior of liquids in moving containers, 1966 NASA SP-106, Washington, DC.

[16] M. Aslam, W.G. Godden and D.T. Scalise, Earthquake sloshing in annular and cylindrical tanks, Journal of the Engineering Mechanics Division 105(3) (1979), 371-389.

[17] M. Aslam, Finite element analysis of earthquake induced sloshing in axisymmetric tanks, International Journal for Numerical Methods 17 (1981), 159-169. 
[18] H.F. Bauer, Forced liquid oscillations in paraboloid containers, Zeitschrift fuer Flugwissenschaften und Weltraumforschung 8 (1984), 49-55.

[19] H.F. Bauer and W. Eidel, Liquid oscillations in a prolate spheroidal container, Archive of Applied Mechanics 59(5) (1989), 371-381

[20] B. Budiansky, Sloshing of liquids in circular canals and spherical tanks, Journal of Aerospace Science 27(3) (1960), 161-173.

[21] M. Isaacson and C.S. Ryu, Earthquake-induced sloshing in vertical container of arbitrary section, Journal of the Engineering Mechanics Division 124(2) (1998), 158-166.

[22] N. Kobayashi, T. Mieda, H. Shibata and Y. Shinozaki, A study of the liquid sloshing response in horizontal cylindrical tanks, Journal of Pressure Vessel Technology 111 (1989), 32-38.

[23] S. Papaspyrou, D. Valougeorgis and S.A. Karamanos, Refined solutions of externally induced sloshing in half-full spherical containers, Journal of Engineering Mechanics 129(12) (2003), 1369-1379.

[24] S. Papaspyrou, S.A. Karamanos and D. Valougeorgis, Response of half-full horizontal cylinders under transverse excitation, Journal of Fluids and Structures 19 (2004), 985-1003.

[25] A. Gedikli and M.E. Ergüven, Seismic analysis of a liquid storage tank with a baffle, Journal of Sound and Vibration 223(1) (1999), $141-155$.

[26] A. Maleki and M. Ziyaeifar, Sloshing damping in cylindrical liquid storage tanks with baffles, Journal of Sound and Vibration 311(2) (2008), 372-385

[27] K.C. Biswal, S.K. Bhattacharyya and P.K. Sinha, Free vibration analysis of liquid filled tank with baffles, Journal of Sound and Vibration 259(1) (2003), 177-192.

[28] K.C. Biswal, S.K. Bhattacharyya and P.K. Sinha, Dynamic response analysis of a liquid-filled cylindrical tank with annular baffle, Journal of Sound and Vibration 274(1) (2004), 13-37.

[29] R.D. Firouz-Abadi, H. Haddadpour, M.A. Noorian and M. Ghasemi, A 3D BEM model for liquid sloshing in baffled tanks, International Journal for Numerical Methods in Engineering 76(9) (2008), 1419-1433.

[30] R.D. Firouz-Abadi, H. Haddadpour and M. Ghasemi, Reduced order modeling of liquid sloshing in 3D tanks using boundary element method, Engineering Analysis with Boundary Elements 33(6) (2009), 750-761.

[31] J.D. Wang, D. Zhou and W.Q. Liu, Sloshing of liquid in rigid cylindrical container with a rigid annular baffle. Part I: Free vibration, Shock and Vibration, accepted for publication, 2012 

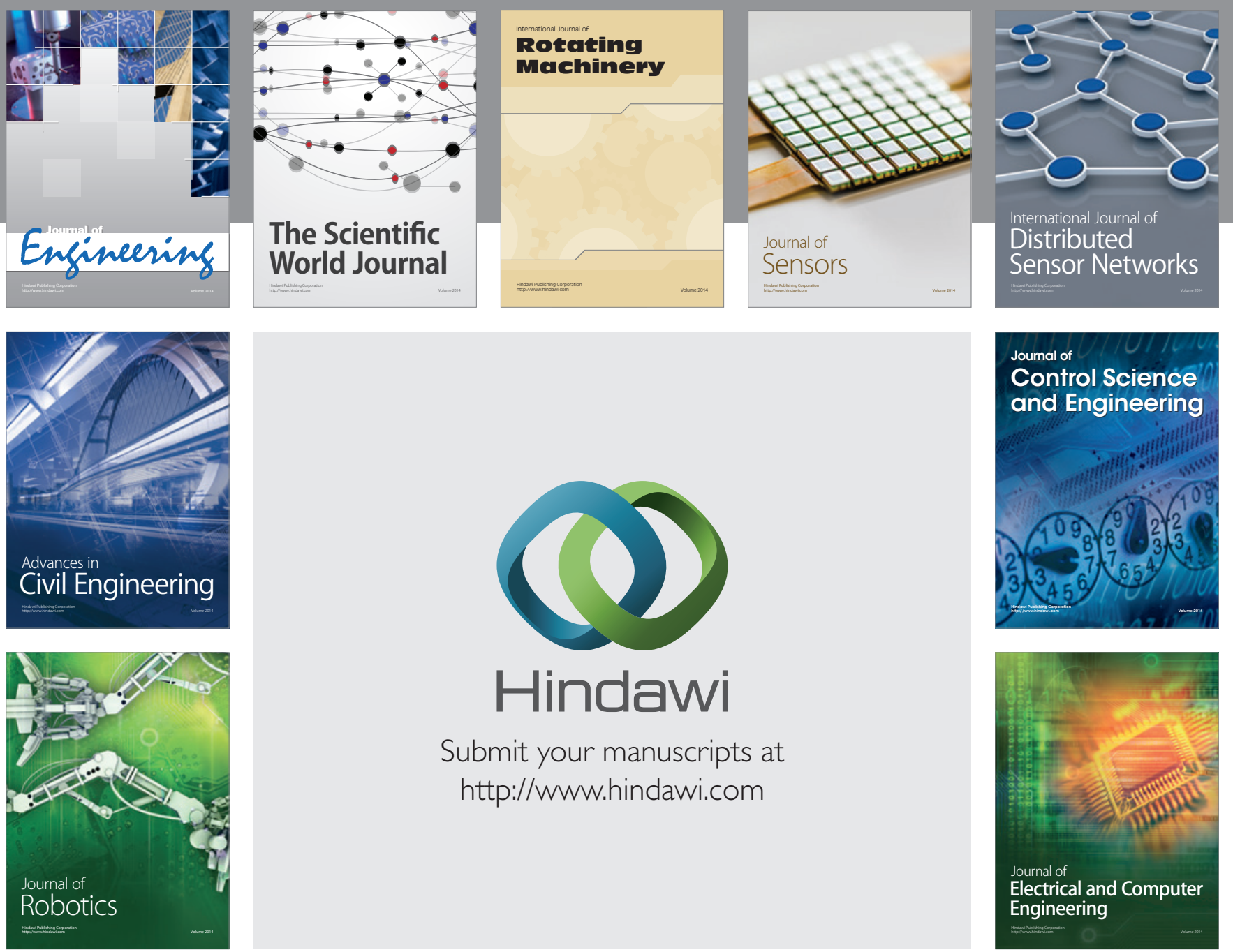

Submit your manuscripts at

http://www.hindawi.com
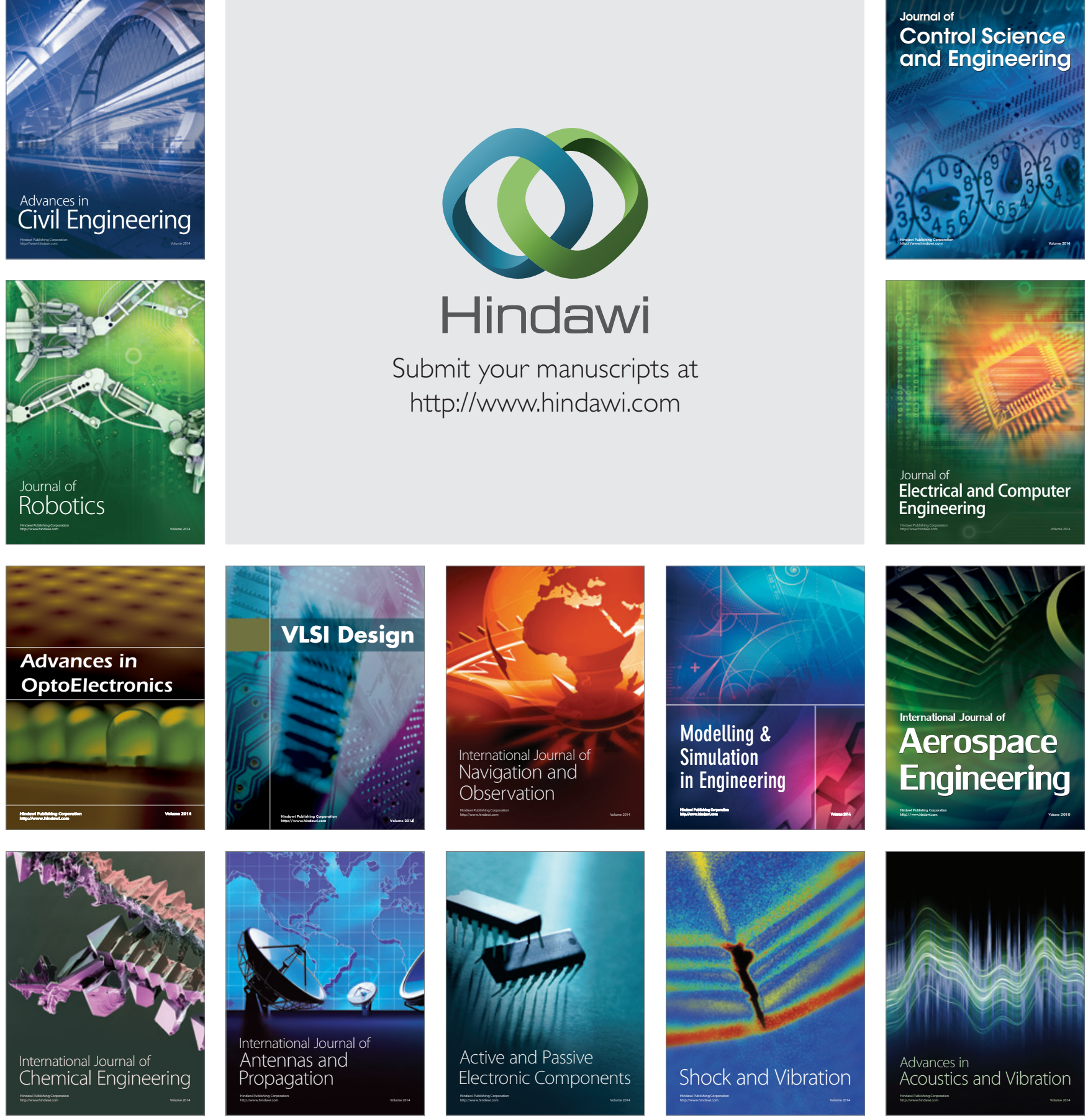